\title{
LINEAR RESPONSE FOR INTERMITTENT MAPS
}

\author{
VIVIANE BALADI AND MIKE TODD
}

\begin{abstract}
We consider the one parameter family $\alpha \mapsto T_{\alpha}(\alpha \in[0,1))$ of PomeauManneville type interval maps $T_{\alpha}(x)=x\left(1+2^{\alpha} x^{\alpha}\right)$ for $x \in[0,1 / 2)$ and $T_{\alpha}(x)=2 x-1$ for $x \in[1 / 2,1]$, with the associated absolutely continuous invariant probability measure $\mu_{\alpha}$. For $\alpha \in(0,1)$, Sarig and Gouëzel proved that the system mixes only polynomially with rate $n^{1-1 / \alpha}$ (in particular, there is no spectral gap). We show that for any $\psi \in L^{q}$, the map $\alpha \rightarrow \int_{0}^{1} \psi d \mu_{\alpha}$ is differentiable on $[0,1-1 / q)$, and we give a (linear response) formula for the value of the derivative. This is the first time that a linear response formula for the SRB measure is obtained in the setting of slowly mixing dynamics. Our argument shows how cone techniques can be used in this context. For $\alpha \geq 1 / 2$ we need the $n^{-1 / \alpha}$ decorrelation obtained by Gouëzel under additional conditions.
\end{abstract}

\section{INTRODUCTION}

Given a family of dynamical systems $T_{\alpha}$ on a Riemann manifold, depending smoothly on a real parameter $\alpha$, and admitting (at least for some large subset of parameters) an ergodic physical (e.g. absolutely continuous, or SRB) invariant measure $\mu_{\alpha}$, it is natural to ask how smooth is the dependence of $\mu_{\alpha}$ on the parameter $\alpha$. In particular, one would like to know whether $\alpha \mapsto \mu_{\alpha}$ is differentiable and, if possible, compute a formula for the derivative, depending on $\mu_{\alpha}, T_{\alpha}$, and $v_{\alpha}=\partial_{\alpha} T_{\alpha}$.

This theme of linear response was explored in a few pioneering papers [Ru1, KKPW, $\mathrm{Ru}]$ in the setting of smooth hyperbolic dynamics (Anosov or Axiom A), and then further developed, following the influence of ideas of David Ruelle. In the smooth hyperbolic case, the SRB measure $\mu_{\alpha}$ corresponds to the fixed point of a transfer operator $\mathcal{L}_{\alpha}$ enjoying a spectral gap on a suitable Banach space. In particular, this fixed point is a simple isolated eigenvalue in the spectrum of $\mathcal{L}_{\alpha}$, and linear response can be viewed as an instance of perturbation theory for simple eigenvalues. This is evident in the linear response formulas, which all involve some avatar of the resolvent $\left(\mathrm{id}-\mathcal{L}_{\alpha}\right)^{-1}=\sum_{k} \mathcal{L}_{\alpha}^{k}$ applied to a suitable vector $Y_{\alpha}$, depending on the derivative of $\mu_{\alpha}$ and on $v_{\alpha}$.

It was soon realised that existence of a spectral gap is not sufficient to guarantee linear response when bifurcations are present (see e.g. [Ma, B1, BS]). In the other direction,

This work was started in 2014 during a visit of MT to DMA-ENS, continued during a visit of VB to St Andrews in 2015, and finished during a stay of VB in the Centre for Mathematical Sciences in Lund. We are grateful to these institutions for their hospitality, and we thank I. Melbourne for pointing out reference [Th2] and A. Korepanov for inciting us to sharpen our results. VB thanks H. Bruin for explanations on [BT] and T. Persson for a conversation on $L^{q}$. She is much indebted to S. Gouëzel for pointing out Theorem 2.4.14 in Goth, which allowed us to extend our results to $\alpha \in[1 / 2,1)$. 
neither the spectral gap nor structural stability is necessary for linear response, as was shown by Dolgopyat [Do] who obtained a linear response formula for some rapidly mixing systems (which were not all exponentially mixing or structurally stable).

The intuition that a key sufficient condition is convergence of the sum $\sum_{k} \mathcal{L}_{\alpha}^{k}\left(Y_{\alpha}\right)$ was confirmed by [HM, Remark 2.4]. This is of course related to a summable decay of correlations. However, decay of correlation usually only holds for observables with a suitable modulus of continuity, which $Y_{\alpha}$, being a derivative, does not always enjoy. We confirm this intuition by studying a toy-model, of Pomeau-Manneville type: 11 For $\alpha \in[0,1)$, we consider the maps (as in [LSV]) $T_{\alpha}:[0,1] \rightarrow[0,1]:$

$$
T_{\alpha}(x)= \begin{cases}x\left(1+2^{\alpha} x^{\alpha}\right), & x \in[0,1 / 2) \\ 2 x-1, & x \in[1 / 2,1]\end{cases}
$$

(Of course, $T_{0}$ is just the angle-doubling map $T_{0}(x)=2 x$ modulo 1 .) It is well-known that each such $T_{\alpha}$ admits a unique absolutely continuous invariant probability measure $\mu_{\alpha}=\rho_{\alpha} d x$. (Clearly, $\rho_{0}(x) \equiv 1$.) Statistical stability (continuity) of $\mu_{\alpha}$ when $\alpha$ changes is proved in [FT]. The absolutely continuous invariant probability measure $\mu_{\alpha}=\rho_{\alpha} d x$ is mixing for all $\alpha \in[0,1)$. For $\alpha=0$ the mixing rate for Lipschitz observables, say, is exponential (decaying like $1 / 2^{k}$ ). For $\alpha \in(0,1)$ the mixing rate is only polynomial with rate $n^{1-1 / \alpha}$ Go, Sa]. (In fact, Gouëzel obtains a faster rate $n^{-1 / \alpha}$ for $\int\left(\psi \circ T_{\alpha}^{n}\right) \phi d \mu_{\alpha}$, if $\psi$ is bounded, $\phi$ is Lipschitz and vanishes in a neighbourhood of zero, and $\int \phi d \mu_{\alpha}=0$, and this property is crucial below when $\alpha \geq 1 / 2$.) In particular, for any $\alpha \in(0,1)$, the density $\rho_{\alpha}$ cannot be the fixed point of a transfer operator with a spectral gap on a Banach space containing all $\mathcal{C}^{\infty}$ functions. However, we are able to prove (Theorem 2.1) that for any $q \in[1, \infty]$ and any $\psi \in L^{q}$, the map $\alpha \mapsto \int \psi d \mu_{\alpha}$ is continuously differentiable on $[0,1-1 / q)$, and we give two expressions (2.6), with a resolvent, 2.7), of susceptibility function type) for the linear response formula, with $Y_{\alpha}=\left(X_{\alpha} \mathcal{N}_{\alpha}\left(\rho_{\alpha}\right)\right)^{\prime}$, where $X_{\alpha}=v_{\alpha} \circ\left(\left.T_{\alpha}\right|_{[0,1 / 2)} ^{-1}\right)$ and $\mathcal{N}_{\alpha}$ corresponds to the first branch of the transfer operator $\mathcal{L}_{\alpha}$. This is the first time that a linear response formula is achieved for a slowly mixing dynamics. The fact that linear response holds for any bounded $\psi$ is relevant since nonsmooth observables appear naturally. For example, if $A$ is smooth and $\Theta$ is a Heaviside function, the expectation value of $\Theta(A(x))$ gives the fraction of the total measure where $A$ has positive value, and more generally such discontinuous observables have probabilistic and physical interpretations, with the work of Lucarini et al. [Lu1, Lu2] showing how the theory of extremes for dynamical systems (in particular regarding climate change) can be cast in this framework.

Our proof is based on the cone techniques from [LSV], and hinges on the new observation that the factor $X_{\alpha}$, respectively $X_{\alpha}^{\prime}$, compensates the singularity at zero of $\rho_{\alpha}^{\prime}$, respectively $\rho_{\alpha}$. Indeed, the compensation is drastic enough so that the $n^{-1 / \alpha}$ decorrelation results of Gouëzel [Go, Goth] can be used. It would apply e.g. to the more general one-dimensional maps with finitely many neutral points described in [LSV, Section 5]. Since our goal is to

\footnotetext{
${ }^{1}$ See Remark 2.2 for one possible generalisation.
} 
describe a new mechanism (demonstrating in particular how invariant cone techniques ${ }^{2}$ can be implemented) for linear response in the presence of neutral fixed points in the simplest setting, we leave such generalisations to further works.

We end this introduction with comments about bifurcations and the singularities of $\rho_{\alpha}$.

By [Th1, Theorem 1], for any $\alpha \in(0,1)$, there exists $0<c_{1}<c_{2}$ so that ${ }^{3}$

$$
c_{1} x^{-\alpha} \leq \rho_{\alpha}(x) \leq c_{2} x^{-\alpha} .
$$

It is easy to see (e.g. via symbolic dynamics) that the maps $\left\{T_{\alpha} \mid \alpha \in[0,1)\right\}$ belong to the same topological class, so that bifurcations do not occur. However the conjugacy $h_{\alpha}$ between $T_{0}$ and $T_{\alpha}$ is not differentiable. Indeed, if it were, then we would have $T_{\alpha}^{\prime}(0)=T_{0}^{\prime}(0)$ at the fixed point 0 , but this is impossible ${ }^{4}$ since $T_{0}^{\prime}(0)=2$, while $T_{\alpha}^{\prime}(0)=1$ for $\alpha>0$. More generally, take arbitrary $\alpha \neq \beta$. The conjugacy $h_{\beta, \alpha}$ between $T_{\alpha}$ and $T_{\beta}$ maps the invariant density $\rho_{\alpha}$ to $\rho_{\beta}$. Therefore $h_{\beta, \alpha}$ cannot be differentiable, since otherwise it would contradict 1.1.

Another lesson of recent research [Ru2, $\mathrm{Ru} 3, \mathrm{~B} 3, \mathrm{~B} 2, \mathrm{CD}]$ on linear response is that understanding the singularities of the SRB measure is essential. In our application, the density $\rho_{\alpha}$ is smooth on $(0,1]$. The only "critical point" of $T_{\alpha}$ is the neutral fixed point at 0 , so that the "postcritical orbit" is reduced to a single point. By (1.1), the singularity type of $\rho_{\alpha}$ at 0 is $x^{-\alpha}$. So, heuristically, for a bounded observable $\psi$, the contribution of the origin to $\partial_{\alpha} \int \psi d \mu_{\alpha}$ should be $\int x^{-\alpha} \log x \cdot \psi(x) d x$, which is indeed well defined $5^{5}$ Indeed, this heuristic remark sheds some light on the otherwise mysterious singularity cancellation $X_{\alpha} \rho_{\alpha}^{\prime} \sim \log x \sim X_{\alpha}^{\prime} \rho_{\alpha}$. Our approach should extend to give higher order derivatives of $\alpha \rightarrow \mu_{\alpha}$ (using invariant cones with more derivatives).

After the first version of this paper (in which our result was restricted to $\alpha \in[0,1 / 2$ ) and $L^{\infty}$ observables) was posted on the arXiv, Korepanov [Ko obtained linear response (without the formula) for all $\alpha \in(0,1)$ and $L^{q}$ observables (for $q>(1-\alpha)^{-1}$ ). His method of proof (using inducing) is different from ours.

\section{Linear Response formula for Pomeau-Manneville maps}

2.1. Statement of the main result. We consider the transfer operator $\mathcal{L}_{\alpha}$ defined, e.g. on $L^{\infty}(d x)$, by (note that $\inf T_{\alpha}^{\prime} \geq 1$ so absolute values are not needed)

$$
\mathcal{L}_{\alpha} \varphi(x)=\sum_{T_{\alpha}(y)=x} \frac{\varphi(y)}{T_{\alpha}^{\prime}(y)} .
$$

\footnotetext{
${ }^{2}$ Bomfim et al. BCV use invariant cones to obtain differentiability of some equilibrium measures (enjoying a spectral gap) of Pomeau-Manneville maps. Their results do not apply to the SRB measure, and thus do not include linear response in the sense of the present work.

${ }^{3}$ The upper bound also follows from [LSV Lemma 2.3].

${ }^{4}$ Also, there are many periodic points $x_{0}$ for $T_{0}$ such that if $p$ is the period then $\left(T_{0}^{p}\right)^{\prime}\left(x_{0}\right)=2^{p}$, but $\left(T_{\alpha}^{p}\right)^{\prime}\left(h_{\alpha}\left(x_{0}\right)\right) \neq 2^{p}$.

${ }^{5}$ While this paper was being finished, it was pointed out to us by I. Melbourne that this heuristic argument can be made rigorous for the special family of maps studied by Thaler in [Th2, Section 2], where the invariant density takes an explicit form.
} 
(For functions depending both on $\alpha$ and $x$ we denote by ' the derivative w.r.t. $x$ and $\partial_{\alpha}$ the derivative w.r.t. $\alpha$.)

We introduce some notation in order to state our main result. Let $f_{\alpha}:[0,1 / 2] \rightarrow[0,1]$ and $g_{\alpha}:[0,1] \rightarrow[0,1 / 2]$ be defined by

$$
f_{\alpha}(x)=x\left(1+2^{\alpha} x^{\alpha}\right), \quad g_{\alpha}(y)=f_{\alpha}^{-1}(y) .
$$

Note that $g_{0}(y)=y / 2$, while for $\alpha>0$ we have

$$
\left|g_{\alpha}(y)-y\left(1-2^{\alpha} y^{\alpha}\right)\right| \leq C\left(y^{1+2 \alpha}\right), \forall y \in[0,1] .
$$

For $0 \leq x \leq 1 / 2$ and $0 \leq \beta \leq 1$, we have $v_{\beta}(x):=\partial_{\beta} T_{\beta}(x)=2^{\beta} x^{1+\beta} \log (2 x)$. Therefore, for $0 \leq x \leq 1$ and $0 \leq \beta \leq 1$,

$$
X_{\beta}:=v_{\beta} \circ g_{\beta}=2^{\beta} g_{\beta}^{1+\beta} \log \left(2 g_{\beta}\right), \quad\left|X_{\beta}(x)\right| \leq c x^{1+\beta}(|\log x|+1),
$$

and

$$
X_{\beta}^{\prime}=2^{\beta} g_{\beta}^{\prime} g_{\beta}^{\beta}\left[(1+\beta) \log \left(2 g_{\beta}\right)+1\right], \quad \quad\left|X_{\beta}^{\prime}(x)\right| \leq c x^{\beta}(|\log x|+1),
$$

and, finally,

$$
\begin{gathered}
X_{\beta}^{\prime \prime}=2^{\beta} g_{\beta}^{\beta-1}\left[g_{\beta}^{\prime \prime} g_{\beta}\left((1+\beta) \log \left(2 g_{\beta}\right)+1\right)+\left(g_{\beta}^{\prime}\right)^{2}\left(\left(\beta+\beta^{2}\right) \log \left(2 g_{\beta}\right)+1+2 \beta\right)\right], \\
\left|X_{\beta}^{\prime \prime}(x)\right| \leq c x^{\beta-1}(|\log x|+1) .
\end{gathered}
$$

(The properties of $X_{\beta}$ and its derivatives above are at the heart of the mechanism of the proofs.) Since $T_{\alpha}(x)$ is independent from $\alpha$ if $x>1 / 2$, to state our main result, we need the transfer operator associated to the first branch of $T_{\alpha}$ by

$$
\mathcal{N}_{\alpha} \varphi(x)=g_{\alpha}^{\prime}(x) \cdot \varphi\left(g_{\alpha}(x)\right) \text {. }
$$

Theorem 2.1 (Linear response formula). Let $\alpha \in(0,1)$. Then for any $q>(1-\alpha)^{-1}$ and any $\psi \in L^{q}(d x)$

$$
\lim _{\epsilon \rightarrow 0} \epsilon^{-1}\left(\int_{0}^{1} \psi d \mu_{\alpha}-\int_{0}^{1} \psi d \mu_{\alpha+\epsilon}\right)=-\int_{0}^{1} \psi\left(\mathrm{id}-\mathcal{L}_{\alpha}\right)^{-1}\left[\left(X_{\alpha} \mathcal{N}_{\alpha}\left(\rho_{\alpha}\right)\right)^{\prime}\right] d x .
$$

(In particular the right-hand side of $(2.6)$ is well-defined.) In addition, the right-hand side of 2.6 can be written as the following absolutely convergent sum

$$
-\sum_{k \geq 0} \int_{0}^{1} \psi \mathcal{L}_{\alpha}^{k}\left[\left(X_{\alpha} \mathcal{N}_{\alpha}\left(\rho_{\alpha}\right)\right)^{\prime}\right] d x=-\sum_{k \geq 0} \int_{0}^{1}\left(\psi \circ T_{\alpha}^{k}\right)\left(X_{\alpha} \mathcal{N}_{\alpha}\left(\rho_{\alpha}\right)\right)^{\prime} d x .
$$

The result also holds for $\alpha=0$, taking the limit as $\epsilon \downarrow 0$ in 2.6 . For $p \in[1, \infty)$, the map $\alpha \mapsto \partial_{\alpha} \rho_{\alpha} \in L^{p}(d x)$ is continuous on $[0,1 / p)$.

Integration by parts allows us to rewrite the convergent sum as

$$
-\sum_{k \geq 0} \int_{0}^{1}\left(\psi \circ T_{\alpha}^{k}\right)\left(X_{\alpha} \mathcal{N}_{\alpha}\left(\rho_{\alpha}\right)\right)^{\prime} d x=\sum_{k \geq 0} \int_{0}^{1}\left(\psi \circ T_{\alpha}^{k}\right)^{\prime} X_{\alpha} \mathcal{N}_{\alpha}\left(\rho_{\alpha}\right) d x .
$$

We conjecture that the above results also hold for $\alpha<0$ in some parameter range, but the proof will require modifications. 
Remark 2.2. It seems our proof also applies to the one-parameter family

$$
G_{t}(x)=\left\{\begin{array}{ll}
(1+t) x+(1-t) 2^{t} x^{1+t}, & x \in[0,1 / 2), \\
2 x-1, & x \in[1 / 2,1],
\end{array} \quad t \in[0, \epsilon] .\right.
$$

Remark 2.3. For the sake of comparison with previous works (e.g. [B3]), we can consider a one-parameter family $F_{\beta}$ obtained by perturbations in the image, i.e. so that $v_{\beta}=\partial_{\beta} F_{\beta}=$ $X_{\beta} \circ F_{\beta}$ for some $X_{\beta}$. This can be achieved by perturbing the first branch $(x<1 / 2)$ in order to have $T_{\beta}=F_{\beta}$ and requiring the second branch $(x \geq 1 / 2)$ to move "sympathetically" with the first one. More precisely, for fixed $\alpha$, consider the one-parameter family $F_{\beta, \alpha}$ which satisfies

$$
F_{\alpha, \alpha}(x)=T_{\alpha}(x), \forall x \in[0,1], \quad F_{\beta, \alpha}(x)=T_{\beta}(x), \forall x \in[0,1 / 2),
$$

and, setting

$$
X_{\beta}(x)=: v_{\beta} \circ g_{\beta}(x)=\partial_{\beta} T_{\beta} \circ g_{\beta}(x), \forall x \in[0,1],
$$

so that

$$
v_{\beta, \alpha}:=\partial_{\beta} F_{\beta, \alpha}=X_{\beta} \circ F_{\beta, \alpha} .
$$

(For $x \in[0,1 / 2)$ this is automatic, and for $x \in[1 / 2,1]$ it can be obtained by solving the ODE $\partial_{\beta} F_{\beta, \alpha}=X_{\beta} \circ F_{\beta, \alpha}$ with initial condition $F_{\alpha, \alpha}(x)=2 x-1$ on $[1 / 2,1]$. By the PicardLindelöf theorem, this ODE has a unique solution since $(\beta, y) \mapsto X_{\beta}(y)$ is continuous in $\beta$.) If $\alpha$ is fixed, slightly abusing notation, we sometimes write $F_{\beta}, v_{\beta}, X_{\beta}$, and $\mathcal{L}_{\beta}$, instead of $F_{\beta, \alpha}, v_{\beta, \alpha}, X_{\beta, \alpha}$, and $\mathcal{L}_{\beta, \alpha}$, when the meaning is clear. It is not difficult to prove that $F_{\beta}$ has a unique absolutely continuous invariant measure $\hat{\mu}_{\beta}=\hat{\rho}_{\beta} d x$ satisfying the same properties as $\mu_{\beta}$, and the proof of Theorem 2.1 shows that for any $\alpha \in(0,1)$ and any $\psi \in L^{\infty}(d x)$

$$
\lim _{\epsilon \rightarrow 0} \epsilon^{-1}\left(\int_{0}^{1} \psi d \hat{\mu}_{\alpha}-\int_{0}^{1} \psi d \hat{\mu}_{\alpha+\epsilon}\right)=-\int_{0}^{1} \psi\left(\mathrm{id}-\mathcal{L}_{\alpha}\right)^{-1}\left[\left(X_{\alpha} \hat{\rho}_{\alpha}\right)^{\prime}\right] d x .
$$

The result also holds for $\alpha=0$, taking the limit as $\epsilon \downarrow 0$ in 2.8 .

Just like (2.6), the expression (2.8) can be written as an absolutely convergent sum. Integration by parts gives $\int\left(\psi^{\prime} \circ T_{\alpha}^{k}\right) \cdot\left(T_{\alpha}^{k}\right)^{\prime}(x) X_{\alpha}(x) \rho_{\alpha}(x) d x$. This is just $\Psi(1)$ where $\Psi(z)$ is the susceptibility function (see e.g. [B3]). It would be interesting to analyse the singularity type of the susceptibility function at $z=1$. (See [BMS] for the corresponding analysis for piecewise expanding maps.)

2.2. Invariant cones. Before, proving the theorem, we introduce notations and state useful results regarding cones adapted from [LSV].

As our proof requires higher derivatives we shall use the following fact:

Proposition 2.4 (Invariant cone in $\mathcal{C}^{2}$ ). For fixed $b_{1} \geq \alpha+1, b_{2} \geq b_{1}, \bar{b}_{1}>0, \bar{b}_{2}>0$, define the cone $\mathcal{C}_{2}$ to be the set of $\varphi \in \mathcal{C}^{2}(0,1]$ so that

$$
\varphi(x) \geq 0, \quad \frac{\bar{b}_{1}}{x} \varphi(x) \leq-\varphi^{\prime}(x) \leq \frac{b_{1}}{x} \varphi(x), \text { and } \frac{\bar{b}_{2}}{x^{2}} \varphi(x) \leq \varphi^{\prime \prime}(x) \leq \frac{b_{2}}{x^{2}} \varphi(x), \forall x \in(0,1] .
$$


Then there exists $b_{\max }<\infty$ so that for any $0 \leq \alpha<1$ there exists $\alpha+1 \leq b^{\prime}(\alpha)<b_{\max }$ and $\bar{b}>1 / b_{\max }$ so that if $b_{1} \geq \alpha+1, b_{2} \geq b^{\prime}(\alpha), \max \left\{\bar{b}_{1}, \bar{b}_{2}\right\} \leq 1 / b^{\prime}(\alpha)$ we have

$$
\varphi \in \mathcal{C}_{2} \Longrightarrow \mathcal{L}_{\alpha}(\varphi) \in \mathcal{C}_{2} \text { and } \mathcal{N}_{\alpha}(\varphi) \in \mathcal{C}_{2} \text {. }
$$

The proof of Proposition 2.4 is given in Appendix A.

For $\varphi \in L^{1}(d x)$ we set $m(\varphi)=\int_{0}^{1} \varphi(x) d x$. For $a \geq 1$, we denote by $\mathcal{C}_{*}=\mathcal{C}_{*}(\alpha, a)$ the cone

$$
\mathcal{C}_{*}:=\left\{\varphi \in \mathcal{C}^{1}(0,1] \mid 0 \leq \varphi(x) \leq 2 a \rho_{\alpha}(x) m(\varphi),-\frac{\alpha+1}{x} \varphi(x) \leq \varphi^{\prime}(x) \leq 0, \forall x \in(0,1]\right\} .
$$

By [LSV, Lemma 2.2], we have ${ }^{6}$

$$
\mathcal{L}_{\alpha}\left(C_{*}(\alpha, a)\right) \subset \mathcal{C}_{*}(\alpha, a), \quad \forall a \geq 2^{\alpha}(\alpha+2) .
$$

Note also that by definition (this will be used to show (2.17))

$$
\int_{0}^{1 / 2} \varphi d x \geq \frac{1}{2} m(\varphi), \quad \forall \varphi \in \mathcal{C}_{*} .
$$

Finally, for $a \geq 2^{\alpha}(\alpha+2)$ and $b_{1} \geq \alpha+1$, we denote by $\mathcal{C}_{*, 1}=\mathcal{C}_{*, 1}\left(\alpha, a, b_{1}\right)$ the cone

$$
\mathcal{C}_{*, 1}:=\left\{\varphi \in \mathcal{C}^{1}(0,1]\left|0 \leq \varphi(x) \leq 2 a \rho_{\alpha}(x) m(\varphi),\right| \varphi^{\prime}(x) \mid \leq \frac{b_{1}}{x} \varphi(x), \forall x \in(0,1]\right\} .
$$

Note that 1.1 implies

$$
\varphi(x) \leq \frac{2 a c_{2}}{x^{\alpha}} m(\varphi), \quad \forall \varphi \in \mathcal{C}_{*, 1}(\alpha), \forall x \in(0,1] .
$$

By definition, $\mathcal{C}_{*, 1} \subset L^{1}(d x)$, and

$$
\beta \geq \alpha \geq 0 \Longrightarrow \mathcal{C}_{*, 1}\left(\alpha, a, b_{1}\right) \subset \mathcal{C}_{*, 1}\left(\beta, \frac{c_{2}}{c_{1}} a, b_{1}\right)
$$

Also, the arguments of [LSV] give $a^{\prime}(\alpha)$ so that

$$
\rho_{\alpha} \in \mathcal{C}_{*}(\alpha) \cap \mathcal{C}_{*, 1}(\alpha) \cap \mathcal{C}_{2}
$$

if the parameters satisfy $a \geq a^{\prime}(\alpha), b_{1} \geq \alpha+1$, and $b_{2}>b^{\prime}(\alpha)$.

Remark 2.5 (Cones $\mathcal{C}_{*}$ and $\mathcal{C}_{*, 1}$ ). The definition of $\mathcal{C}_{*, 1}$ will make it easy to check that $-\left(X_{\alpha} \varphi\right)^{\prime}+c_{\varphi}$, for suitable $\varphi \in \mathcal{C}_{*, 1}$ and constant $c_{\varphi}>0$, lies in a cone $\mathcal{C}_{*, 1}$ (possibly for larger $a$ and $b_{1}$ ), via the Leibniz formula. In $\$ 2.3$ this will be used to get bounds $\varphi(x) \leq C x^{-\alpha}$ and $\left|\varphi^{\prime}(x)\right| \leq C x^{-1-\alpha}$, while in Appendix B these cones play a more important role. This is why we use $\mathcal{C}_{*, 1}$ instead of the cone $\mathcal{C}_{*}$ used in [LSV]. However, the condition that $\varphi(x)$ is decreasing will be used to get (2.17). (In [LSV] the condition that $\varphi(x)$ be decreasing is only used in [LSV, Lemma 2.1], which we do not need in view of Proposition 2.4 and (B.1).)

We will use the following result (see Appendix A for the proof):

\footnotetext{
${ }^{6}$ Noting that $-\frac{\alpha+1}{x} \varphi(x) \leq \varphi^{\prime}(x) \leq 0$ if and only if $\varphi$ is decreasing and $x^{\alpha+1} \varphi$ is increasing.
} 
Proposition 2.6 (Invariance of the cone $\mathcal{C}_{*, 1}$ ). Fix $\alpha \in(0,1)$, $a \geq 1$, and $b_{1} \geq \alpha+1$. Then

$$
\begin{aligned}
& \mathcal{L}_{\alpha}\left(\mathcal{C}_{*, 1}\left(\alpha, a, b_{1}\right)\right) \subset \mathcal{C}_{*, 1}\left(\alpha, a, b_{1}\right), \\
& \mathcal{N}_{\alpha}\left(\mathcal{C}_{*, 1}\left(\alpha, a, b_{1}\right) \cap\left\{\int_{0}^{1 / 2} \varphi d x \geq \frac{1}{2} m(\varphi)\right\}\right) \subset \mathcal{C}_{*, 1}\left(\alpha, 2 a, b_{1}\right) .
\end{aligned}
$$

In addition, there exists $C>0$, independent of $\alpha$, $a$, and $b_{1}$ so that we have for any $\psi \in L^{\infty}$ and $\varphi \in \mathcal{C}_{*, 1}(\alpha)+\mathbb{R}$ with $\int \varphi d x=0$,

$$
\left|\int_{0}^{1} \psi \mathcal{L}_{0}^{k}(\varphi) d x\right| \leq \frac{C a b_{1}}{(1-\alpha)(\log k) k^{-2+1 / \alpha}}\|\psi\|_{L^{\infty}}\|\varphi\|_{1}, \forall k \geq 1 .
$$

Note that for fixed $a_{\max }<\infty$ and $b_{\max }<\infty$, the expression 2.18 is controlled by

$$
\sup _{b_{1} \leq b_{\max }, a \leq a_{\max }} \frac{C a b_{1}}{(1-\alpha)(\log k) k^{-2+1 / \alpha}}<\infty .
$$

2.3. Proof of Theorem 2.1. We may now prove the theorem:

Proof of Theorem 2.1. Step 0: We show that the right-hand side of (2.6) is well-defined for bounded $\psi$. First observe that integration by parts and $X_{\alpha}(0)=X_{\alpha}(1)=0$ (because $v_{\alpha}(0)=0$ and $\left.v_{\alpha}(1 / 2)=0\right)$ imply

$$
\int_{0}^{1}\left(X_{\alpha} \mathcal{N}_{\alpha}\left(\rho_{\alpha}\right)\right)^{\prime} d x=0
$$

Then note that

$$
\left\|\left(X_{\alpha} \mathcal{N}_{\alpha}\left(\rho_{\alpha}\right)\right)^{\prime}\right\|_{1}=\left\|X_{\alpha}\left(\mathcal{N}_{\alpha}\left(\rho_{\alpha}\right)\right)^{\prime}+X_{\alpha}^{\prime} \mathcal{N}_{\alpha}\left(\rho_{\alpha}\right)\right\|_{1} \leq C \int_{0}^{1}(|\log x|+1) d x<\infty .
$$

Indeed, this is easy for $\alpha=0$ since

$$
X_{0}(x)=\frac{x(\log 2+\log (x / 2))}{2}, \quad X_{0}^{\prime}(x)=\frac{1+\log 2+\log (x / 2)}{2}, \quad \forall x \in[0,1] .
$$

Next, $\left.\rho_{0}\right|_{[0,1]} \equiv 1$, with $\left.\left(\mathcal{N}_{0} \rho_{0}\right)\right|_{[0,1]} \equiv 1 / 2$, so that for any $x \in[0,1]$,

$$
\left(X_{0} \mathcal{N}_{0}\left(\rho_{0}\right)\right)^{\prime}(x)=\frac{X_{0}^{\prime}(x)}{2}=\frac{1+\log 2+\log (x / 2)}{4} .
$$

For $\alpha>0$, on the one hand, (1.1) and Proposition 2.4 imply that

$$
\left|\rho_{\alpha}^{\prime}(x)\right| \leq a c_{2} x^{-1-\alpha}, \mathcal{N}_{\alpha}\left(\rho_{\alpha}\right)(x) \leq \rho_{\alpha}(x) \leq c_{2} x^{-\alpha},\left|\left(\mathcal{N}_{\alpha}\left(\rho_{\alpha}\right)\right)^{\prime}(x)\right| \leq c_{2} b_{1} x^{-1-\alpha} .
$$

On the other hand, the dominant term of $X_{\alpha}(x)$ is a constant multiple of $x^{1+\alpha} \log x$ (see (2.3) while the dominant term of $X_{\alpha}^{\prime}(x)$ is a constant multiple of $x^{\alpha} \log x$ (see (2.4) and recall (2.2)). This establishes (2.20) for $\alpha>0$.

Next, write the right-hand side of (2.6) as

$$
\left|\sum_{j=0}^{\infty} \int_{0}^{1} \psi \cdot \mathcal{L}_{\alpha}^{j}\left[\left(X_{\alpha} \mathcal{N}_{\alpha}\left(\rho_{\alpha}\right)\right)^{\prime}\right] d x\right| \leq \sum_{j=0}^{\infty}\left|\int_{0}^{1} \psi \cdot \mathcal{L}_{\alpha}^{j}\left[\left(X_{\alpha} \mathcal{N}_{\alpha}\left(\rho_{\alpha}\right)\right)^{\prime}\right] d x\right| .
$$


The function $f=-\left(X_{\alpha} \mathcal{N}_{\alpha}\left(\rho_{\alpha}\right)\right)^{\prime} / \rho_{\alpha}$ is Hölder, and it vanishes at zero, with $\int f \rho_{\alpha} d x=0$. In addition, for any $\epsilon \in(0,1)$ we have $C$ so that $|f(x)| \leq C x^{\alpha(1-\epsilon / 2)}$. Since

$$
\frac{1}{\alpha}(1+\alpha(1-\epsilon / 2))-1>\frac{1}{\alpha}-\epsilon,
$$

if $\epsilon>0$ is small enough then [Goth, Thm 2.4.14] applied 7 to $f=-\left(X_{\alpha} \mathcal{N}_{\alpha}\left(\rho_{\alpha}\right)\right)^{\prime} / \rho_{\alpha}$ gives $K_{\alpha}>0$ so that the $j$ th term in the right-hand side of $(2.23)$ is bounded by

$$
\sum_{j=0}^{\infty}\left|\int_{0}^{1}\left(\psi \circ T_{\alpha}^{j}\right) \cdot f d \mu_{\alpha}\right| \leq C K_{\alpha}\|\psi\|_{L^{\infty}} \frac{1}{j^{(1 / \alpha)-\epsilon}} .
$$

Since we may take $\epsilon<(1 / \alpha)-1$, this is summable.

If $\alpha=0$, fixing $\beta>0$, it is easy to see that there exists a constant $C_{X}>0$ so that $-\left(X_{0}^{\prime} \mathcal{N}_{0}\left(\rho_{0}\right)\right)^{\prime}+C_{X}$ belongs to $\mathcal{C}_{*, 1}\left(\beta, a, b_{1}\right)$, for suitable $a$ and $b_{1}$ (see (2.13)). We may apply (2.18) from Proposition 2.6 to $\varphi=-\left(X_{0} \mathcal{N}_{0}\left(\rho_{0}\right)\right)^{\prime} \in \mathcal{C}_{*, 1}+\mathbb{R}$ in order to bound the $j$ th term in the right-hand side of 2.23$)$.

Step 1: Let $\psi$ be a bounded function so that $\int \psi d \mu_{\alpha}=0$. We first show that $\beta \mapsto$ $\int \psi \rho_{\beta} d x$ is Lipschitz at $\beta=\alpha$. Applying the bound on [LSV, p. 680] to $g=\psi$ and the zero-average function $f=\rho_{\alpha}-\mathbf{1} \in \mathcal{C}_{*, 1}+\mathbb{R}$, we have, if $\alpha>0$,

$$
\left|\int_{0}^{1} \psi \circ T_{\alpha}^{k} d x\right|=\left|\int_{0}^{1} \psi \mathcal{L}_{\alpha}^{k}\left(\rho_{\alpha}-1\right) d x\right| \leq C_{\alpha}\|\psi\|_{L^{\infty}} \frac{(\log k)^{1 / \alpha}}{k^{-1+1 / \alpha}},
$$

and, for any $\beta>0$,

$$
\left|\int_{0}^{1} \psi \circ T_{\beta}^{k} d x-\int_{0}^{1} \psi d \mu_{\beta}\right| \leq C_{\beta}\|\psi\|_{L^{\infty}} \frac{(\log k)^{1 / \beta}}{k^{-1+1 / \beta}} .
$$

If $\alpha=0$, then the spectral gap of $\mathcal{L}_{0}$ on $\mathcal{C}^{1}$ e.g. gives a constant $C \geq 1$ so that

$$
\left|\int_{0}^{1} \psi \circ T_{0}^{k} d x\right| \leq C\|\psi\|_{L^{\infty}} 2^{-k}
$$

Taking $k$ large enough, depending on $\beta$ and $\alpha$, the three expressions $2.24-2.25-2.26$ are thus $o(\beta-\alpha)$. More precisely, fixing $\xi>0$, there is $C$ so that, for all

$$
k>C\left(C_{\max (\alpha, \beta)}(\beta-\alpha)^{-(1+\xi)}\right)^{1 /(-1+1 / \max (\alpha, \beta))}
$$

we have

$$
\left|\int_{0}^{1} \psi \circ T_{\alpha}^{k} d x\right|+\left|\int_{0}^{1} \psi \circ T_{\beta}^{k} d x-\int_{0}^{1} \psi d \mu_{\beta}\right| \leq C\|\psi\|_{L^{\infty}}(\beta-\alpha)^{1+\xi} .
$$

\footnotetext{
${ }^{7}$ This theorem is a strengthening of [Go, Prop. 6.11, Cor. 7.1].
} 
Letting 1 be the constant function $\equiv 1$, it thus suffices to bound

$$
\begin{aligned}
\frac{1}{\beta-\alpha}\left(\int_{0}^{1} \psi \circ T_{\beta}^{k} d x-\int_{0}^{1} \psi \circ T_{\alpha}^{k} d x\right) & =\frac{1}{\beta-\alpha} \int_{0}^{1} \psi\left(\mathcal{L}_{\beta}^{k} \mathbf{1}-\mathcal{L}_{\alpha}^{k} \mathbf{1}\right) d x \\
& =\sum_{j=0}^{k-1} \int_{0}^{1} \psi \mathcal{L}_{\beta}^{j}\left(\frac{\mathcal{L}_{\beta}-\mathcal{L}_{\alpha}}{\beta-\alpha}\left(\mathcal{L}_{\alpha}^{k-j-1}(\mathbf{1})\right)\right) d x,
\end{aligned}
$$

uniformly in $\beta \rightarrow \alpha$. For this, we shall use below that for any $\varphi \in \mathcal{C}^{2}(0,1]$, any $x \neq 0$, and any $\beta \neq \alpha$,

$$
\frac{\mathcal{L}_{\beta} \varphi(x)-\mathcal{L}_{\alpha} \varphi(x)}{\beta-\alpha}=\partial_{\alpha} \mathcal{L}_{\alpha} \varphi(x)+\frac{1}{\beta-\alpha} \int_{\alpha}^{\beta} \partial_{\gamma}^{2} \mathcal{L}_{\gamma} \varphi(x)(\gamma-\alpha) d \gamma .
$$

It is easy to check (see the proof of [B3, Thm 2.2]) that we have

$$
\partial_{\alpha} g_{\alpha}(x)=-X_{\alpha}(x) \mathcal{N}_{\alpha}\left(1 / T_{\alpha}^{\prime}\right)(x)=-\frac{X_{\alpha}(x)}{T_{\alpha}^{\prime}\left(g_{\alpha}(x)\right)^{2}},
$$

and, more generally, for any $\varphi \in \mathcal{C}^{1}(0,1]$ and any $x \neq 0$, we have

$$
\partial_{\alpha} \mathcal{L}_{\alpha}(\varphi)(x)=\partial_{\alpha} \mathcal{N}_{\alpha}(\varphi)(x)=\mathcal{M}_{\alpha}(\varphi)(x),
$$

where we set for $x \neq 0$

$$
\begin{aligned}
\mathcal{M}_{\alpha}(\varphi)(x) & =-X_{\alpha}^{\prime} \mathcal{N}_{\alpha}(\varphi)(x)-X_{\alpha} \mathcal{N}_{\alpha}\left(\varphi^{\prime} / T_{\alpha}^{\prime}\right)(x)+X_{\alpha} \mathcal{N}_{\alpha}\left(\varphi T_{\alpha}^{\prime \prime} /\left(T_{\alpha}^{\prime}\right)^{2}\right)(x) \\
& =-\left(X_{\alpha} \cdot \mathcal{N}_{\alpha}(\varphi)\right)^{\prime}(x)
\end{aligned}
$$

Using (2.31) and 2.32) (twice), we also get, for $x \neq 0$ and $\varphi \in \mathcal{C}^{2}(0,1]$,

$$
\begin{aligned}
& \partial_{\alpha}^{2} \mathcal{L}_{\alpha}(\varphi)(x)=-\partial_{\alpha}\left(\left(X_{\alpha} \mathcal{N}_{\alpha}(\varphi)\right)^{\prime}\right)(x) \\
& =-\left(\left(\partial_{\alpha} X_{\alpha}^{\prime}\right)\left(\mathcal{N}_{\alpha}(\varphi)\right)\right)(x)-\left(X_{\alpha}^{\prime}\left(\partial_{\alpha} \mathcal{N}_{\alpha}(\varphi)\right)\right)(x) \\
& \quad-\left(\partial_{\alpha} X_{\alpha}\left(\mathcal{N}_{\alpha}(\varphi)\right)^{\prime}\right)(x)-\left(X_{\alpha} \partial_{\alpha}\left(\mathcal{N}_{\alpha}(\varphi)\right)^{\prime}\right)(x) \\
& =-\left(\left(\partial_{\alpha} X_{\alpha}\right)\left(\mathcal{N}_{\alpha}(\varphi)\right)\right)^{\prime}(x)+X_{\alpha}^{\prime}\left(X_{\alpha} \mathcal{N}_{\alpha}(\varphi)\right)^{\prime}(x)+X_{\alpha}\left(X_{\alpha} \mathcal{N}_{\alpha}(\varphi)\right)^{\prime \prime}(x) .
\end{aligned}
$$

Returning to 2.28), we assume that $\beta>\alpha>0$. For $k \geq 1$, we get, using 2.29 2.32 ,

$$
\begin{aligned}
\sum_{j=0}^{k-1} \int_{0}^{1} \psi \mathcal{L}_{\beta}^{j}\left(\frac{\mathcal{L}_{\beta}-\mathcal{L}_{\alpha}}{\beta-\alpha}\left(\mathcal{L}_{\alpha}^{k-j-1}(\mathbf{1})\right)\right) d x & =-\sum_{j=0}^{k-1} \int_{0}^{1} \psi \mathcal{L}_{\beta}^{j}\left(\left[X_{\alpha} \mathcal{N}_{\alpha}\left(\mathcal{L}_{\alpha}^{k-j-1}(\mathbf{1})\right)\right]^{\prime}\right) d x \\
& +\int_{\alpha}^{\beta} \frac{\gamma-\alpha}{\beta-\alpha} \sum_{j=0}^{k-1} \int_{0}^{1} \psi \mathcal{L}_{\beta}^{j}\left[\partial_{\gamma}^{2} \mathcal{L}_{\gamma}\left(\mathcal{L}_{\alpha}^{k-j-1}(\mathbf{1})\right)\right] d x d \gamma
\end{aligned}
$$

Consider the first term in the right-hand side of (2.34). Observe that $\mathcal{L}_{\alpha} \mathbf{1} \in \mathcal{C}_{*, 1}\left(\alpha, a, b_{1}\right) \cap \mathcal{C}_{2}$, so that, recalling Proposition 2.4 we have that $\mathcal{L}_{\alpha}^{k-j-1}(\mathbf{1})$ is in $\mathcal{C}_{*, 1}(\alpha) \cap \mathcal{C}_{2}$ and thus in $\mathcal{C}_{*, 1}(\gamma) \cap \mathcal{C}_{2}$ for any $\gamma \geq \alpha$ up to increasing $a$ uniformly in $j$ and $k>j-1$. Note that $\left|\left(\mathcal{N}_{\alpha}\left(\mathcal{L}_{\alpha}^{k-j-1}(\mathbf{1})\right)\right)^{\prime}(x)\right| \leq b_{1} c_{2} x^{-1-\alpha}$. Proceeding as in Step 0 (using 2.11) to invoke 2.12) 
to get $(2.17)$ ), we obtain a constant $C>1$ so that $\left|\left[X_{\alpha} \mathcal{N}_{\alpha}\left(\mathcal{L}_{\alpha}^{k-j-1}(\mathbf{1})\right)\right]^{\prime}(x)\right| \leq C(|\log x|+1)$ for all $1 \leq j \leq k-1$ (in particular $\left.\sup _{k} \sup _{1 \leq j \leq k-1}\left\|\left[X_{\alpha} \mathcal{N}_{\alpha}\left(\mathcal{L}_{\alpha}^{k-j-1}(\mathbf{1})\right)\right]^{\prime}\right\|_{1}<\infty\right)$.

Next, if $0<\alpha<\beta<1$, by using [Goth, Thm 2.4.14] (as in Step 0), we get summability of the first term of the expression in the right-hand side of (2.34) as $k \rightarrow \infty$ :

$$
\left|\sum_{j=0}^{k-1} \int_{0}^{1} \psi \mathcal{L}_{\beta}^{j}\left(\left[X_{\alpha} \mathcal{N}_{\alpha}\left(\mathcal{L}_{\alpha}^{k-j-1}(\mathbf{1})\right)\right]^{\prime}\right) d x\right| \leq C_{\beta}\|\psi\|_{L^{\infty}} \sum_{j=0}^{k-1} \frac{1}{j^{(1 / \beta)-\epsilon}} .
$$

Finally, we consider the second term of the right-hand side of (2.34). (This is where we require the derivatives of order two in $\mathcal{C}_{2}$.) Applying $(2.33)$ to $\varphi=\mathcal{L}_{\alpha}^{k-j}-1(\mathbf{1}) \in \mathcal{C}_{2} \cap \mathcal{C}_{*, 1}(\alpha)$, and using Proposition 2.4, we see that for any $\alpha \leq \gamma \leq \beta$,

$$
\left|\left[\partial_{\gamma}^{2} \mathcal{L}_{\gamma}\left(\mathcal{L}_{\alpha}^{k-j-1}(\mathbf{1})\right)\right](x)\right| \leq C(|\log x|+1)^{2} .
$$

Indeed, recalling (2.3) and 2.30), first note that $\left|\partial_{\alpha} X_{\alpha}(x)\right| \leq c x^{1+\alpha}(|\log x|+1)^{2},\left|\partial_{\alpha} X_{\alpha}^{\prime}\right| \leq c x^{\alpha}(|\log x|+1)^{2},\left|\partial_{\alpha} X_{\alpha}^{\prime \prime}\right| \leq c x^{\alpha-1}(|\log x|+1)^{2}$. In addition, for $\varphi$ in $\mathcal{C}_{*, 1}(\alpha) \cap \mathcal{C}_{2}$, we have

$$
\left|\left(\mathcal{N}_{\alpha}(\varphi)\right)^{\prime \prime}(x)\right| \leq \frac{b_{2}}{x^{2}} \rho_{\alpha}(x) m\left(\mathcal{N}_{\alpha}(\varphi)\right) \leq \frac{b_{2} c_{2}}{x^{2+\alpha}} m(\varphi), \quad\left|\left(X_{\alpha} \mathcal{N}_{\alpha}(\varphi)\right)^{\prime \prime}(x)\right| \leq \frac{\hat{c}}{x} .
$$

Labelling the three terms from the right-hand side of 2.33) as $I, I I$, and $I I I$, we expand them via the Leibniz equality, obtaining seven functions:

$$
\begin{aligned}
& I=-\left(\partial_{\alpha} X_{\alpha}\right)^{\prime}\left(\mathcal{N}_{\alpha} \varphi\right)+\partial_{\alpha} X_{\alpha}\left(\mathcal{N}_{\alpha} \varphi\right)^{\prime}, \quad I I=\left(X_{\alpha}^{\prime}\right)^{2} \mathcal{N}_{\alpha}(\varphi)+X_{\alpha}^{\prime} X_{\alpha}\left(\mathcal{N}_{\alpha}(\varphi)\right)^{\prime}, \\
& I I I=X_{\alpha}\left[X_{\alpha}^{\prime \prime} \mathcal{N}_{\alpha}(\varphi)+2 X_{\alpha}^{\prime}\left(\mathcal{N}_{\alpha}(\varphi)\right)^{\prime}+X_{\alpha}\left(\mathcal{N}_{\alpha}(\varphi)\right)^{\prime \prime}\right]
\end{aligned}
$$

By the above, since $\varphi=\mathcal{L}_{\alpha}^{k-j-1}(\mathbf{1}) \in \mathcal{C}_{2} \cap \mathcal{C}_{*, 1}(\alpha)$, we can bound $|I|$ by

$$
\left(c x^{\alpha}(|\log x|+1)^{2}\right)\left(c x^{-\alpha}\right)+\left(\left(c x^{1+\alpha}\right)(|\log x|+1)^{2} \frac{b_{1}}{x} \frac{2 a c_{2}}{x^{\alpha}} m(\varphi)\right) .
$$

Similarly $|I I| \leq\left(c x^{\alpha}(|\log x|+1)(c(|\log x|+1))\right.$ and $|I I I| \leq\left(c x^{1+\alpha}(|\log x|+1)\right) \hat{c} x^{-1}$. This proves (2.36).

Applying [Goth, Thm 2.4.14] once more (as in Step 0) we thus get the bound

$$
\left.\frac{\|\psi\|_{L^{\infty}}}{\beta-\alpha} \int_{\alpha}^{\beta}(\gamma-\alpha) C_{\beta} \sum_{j=0}^{k-1} \frac{1}{j^{(1 / \beta)-\epsilon}}\right) d \gamma \leq C_{\beta}\|\psi\|_{L^{\infty}}(\beta-\alpha) .
$$

If $\alpha \in(0,1)$, the case $\beta<\alpha$ can be handled similarly, substituting

$$
\sum_{j} \mathcal{L}_{\beta}^{j}\left(\mathcal{L}_{\beta}-\mathcal{L}_{\alpha}\right) \mathcal{L}_{\alpha}^{k-j-1}=-\sum_{j} \mathcal{L}_{\alpha}^{j}\left(\mathcal{L}_{\alpha}-\mathcal{L}_{\beta}\right) \mathcal{L}_{\beta}^{k-j-1}
$$

in 2.28), and replacing 2.29 by $\frac{\mathcal{L}_{\alpha} \varphi(x)-\mathcal{L}_{\beta} \varphi(x)}{\alpha-\beta}=\partial_{\beta} \mathcal{L}_{\beta} \varphi(x)+\frac{1}{\alpha-\beta} \int_{\beta}^{\alpha} \partial_{\gamma}^{2} \mathcal{L}_{\gamma} \varphi(x)(\gamma-\beta) d \gamma$.

Finally, if $\alpha=0$, we use $\sum_{j} \mathcal{L}_{\beta}^{j}\left(\mathcal{L}_{\beta}-\mathcal{L}_{0}\right) \mathcal{L}_{0}^{k-j-1}=-\sum_{j} \mathcal{L}_{0}^{j}\left(\mathcal{L}_{0}-\mathcal{L}_{\beta}\right) \mathcal{L}_{\beta}^{k-j-1}$, with 2.29, and exploit 2.18 and 2.19). 
This proves that for $\psi \in L^{\infty}$ the map $\beta \mapsto \int \psi(x) \rho_{\beta}(x) d x$ is locally Lipschitz on $[0,1)$.

Step 2: Still assuming that $\psi$ is bounded, we next prove that $\beta \mapsto \int \psi \rho_{\beta} d x$ is differentiable at $\beta=\alpha \in[0,1)$ and check that the derivative takes the announced value. To prove differentiability, recalling (2.27) and setting $k(\beta)=k(\alpha, \beta, \xi)=C\left(C_{\beta}(\beta-\right.$ $\left.\alpha)^{-(1+\xi)}\right)^{1 /(-1+1 / \max (\alpha, \beta))}$, for some small $\xi>0$, it suffices to check that

$\sum_{j=0}^{k(\beta)} \int_{0}^{1} \psi \mathcal{L}_{\beta}^{j}\left(\left[X_{\alpha} \mathcal{N}_{\alpha}\left(\mathcal{L}_{\alpha}^{k(\beta)-j}(\mathbf{1})\right)\right]^{\prime}\right) d x+\int_{\alpha}^{\beta} \frac{\gamma-\alpha}{\beta-\alpha} \sum_{j=0}^{k(\beta)-1} \int_{0}^{1} \psi \mathcal{L}_{\beta}^{j}\left[\partial_{\gamma}^{2} \mathcal{L}_{\gamma}\left(\mathcal{L}_{\alpha}^{k(\beta)-j-1}(\mathbf{1})\right)\right] d x d \gamma$

converges, when $\beta \rightarrow \alpha>0$ or $\beta \downarrow \alpha=0$, to

$$
\sum_{j=0}^{\infty} \int_{0}^{1} \psi \mathcal{L}_{\alpha}^{j}\left(\left[X_{\alpha} \mathcal{N}_{\alpha}\left(\rho_{\alpha}\right)\right]^{\prime}\right) d x=\int_{0}^{1} \psi \sum_{j=0}^{\infty} \mathcal{L}_{\alpha}^{j}\left(\left[X_{\alpha} \mathcal{N}_{\alpha}\left(\rho_{\alpha}\right)\right]^{\prime}\right) d x
$$

By (2.38), the second term in 2.39) converges to zero as $\beta \rightarrow \alpha$. Next, for $\alpha \in[0,1)$, fixing $\eta>0$ small, by Step 0 we may take $K=K_{\eta}$ large enough so that the $K$-tail of (2.40) is $<\eta / 4$, while the $K$-tail of the first term of 2.39$)$ is $<\eta / 4$ uniformly in $\beta$. It thus suffices, for every fixed $0 \leq j \leq K$, to show that the following difference tends to zero as $\beta \rightarrow \alpha>0$ or $\beta \downarrow 0$ :

$$
\int_{0}^{1}\left[\left(\psi \circ T_{\beta}^{j}\right)\left(\left[X_{\alpha} \mathcal{N}_{\alpha}\left(\mathcal{L}_{\alpha}^{k(\beta)-j}(\mathbf{1})\right)\right]^{\prime}\right)-\left(\psi \circ T_{\alpha}^{j}\right)\left(\left[X_{\alpha} \mathcal{N}_{\alpha}\left(\rho_{\alpha}\right)\right]^{\prime}\right)\right] d x .
$$

So it is sufficient to show that there exists $N_{\eta} \geq 1$ so that

$$
\left\|\left[X_{\alpha} \mathcal{N}_{\alpha}\left(\mathcal{L}_{\alpha}^{k}(\mathbf{1})\right)\right]^{\prime}-\left[X_{\alpha} \mathcal{N}_{\alpha}\left(\rho_{\alpha}\right)\right]^{\prime}\right\|_{L^{1}}<\frac{\eta}{2 K}, \forall k \geq N_{\eta}
$$

If $\alpha=0$, this is easy, since $\rho_{\alpha}=\mathbf{1}$ so that the expression $(2.42)$ vanishes trivially.

If $\alpha \in(0,1)$, setting $\phi_{k}:=\mathcal{L}_{\alpha}^{k}(\mathbf{1})$, we note that the bound on [LSV, p. 680] applied to $g=\mathbf{1}$ and $f=\mathbf{1}-\rho_{\alpha}$ implies $\left\|\phi_{k}-\rho_{\alpha}\right\|_{1} \leq C_{\alpha} k^{1-1 / \alpha}(\log k)^{1 / \alpha}$. (This is not summable if $\alpha \geq 1 / 2$, but it does tend to zero for all $\alpha \in(0,1)$.) Therefore,

$$
\left\|\mathcal{N}_{\alpha}\left(\phi_{k}-\rho_{\alpha}\right)\right\|_{1} \leq C_{\alpha}\left\|\phi_{k+1}-\rho_{\alpha}\right\|_{1} \leq C_{\alpha} \frac{(\log k)^{1 / \alpha}}{k^{-1+1 / \alpha}} .
$$

Since $X_{\alpha}^{\prime} \in L^{\infty}$, it thus suffices to show that

$$
\left\|X_{\alpha}\left[\mathcal{N}_{\alpha}\left(\mathcal{L}_{\alpha}^{k}(\mathbf{1})\right)\right]^{\prime}-X_{\alpha}\left[\mathcal{N}_{\alpha}\left(\rho_{\alpha}\right)\right]^{\prime}\right\|_{L^{1}}<\frac{\eta}{2 K}
$$

For this, first observe that Proposition 2.4 implies that there exists $b_{1}$ so that, for $\varphi=\rho_{\alpha}$ and $\varphi=1$,

$$
\left|\left[\mathcal{N}_{\alpha} \mathcal{L}_{\alpha}^{k}(\varphi)\right]^{\prime}(x)\right| \leq \frac{b_{1}}{x} \varphi(x) \leq \frac{b_{1} a c_{2}}{x^{1+\alpha}}, \forall k \geq 1, \forall x \in(0,1]
$$

Since $\left|X_{\alpha}(z)\right| \leq c_{\alpha}\left|z^{1+\alpha}(\log z+\log 2)\right|$, it follows that there exist $C, \hat{C}>0$ so that for any $\bar{x} \in(0,1]$, all $k \geq 0$, and, for $\varphi=\rho_{\alpha}$ and $\varphi=\mathbf{1}$,

$$
\int_{0}^{\bar{x}}\left|X_{\alpha}(z)\left(\mathcal{N}_{\alpha} \mathcal{L}_{\alpha}^{k}(\varphi)\right)^{\prime}(z)\right| d z \leq C \int_{0}^{\bar{x}}(|\log z|+1) d z \leq \widehat{C} \bar{x}(|\log \bar{x}|+1) .
$$


We will choose $\bar{x}$ to be small and then choose $k$ large enough that the remaining integral is small. We decompose the remaining integral, for $\varphi=\rho_{\alpha}$ and $\varphi=\mathbf{1}$, as

$$
\int_{\bar{x}}^{1} X_{\alpha}(z)\left(\mathcal{N}_{\alpha} \mathcal{L}_{\alpha}^{k}(\varphi)\right)^{\prime}(z) d z=\int_{\bar{x}}^{1} X_{\alpha}(z)\left(\mathcal{L}_{\alpha}^{k+1}(\varphi)\right)^{\prime}(z) d z+\int_{\bar{x}}^{1} X_{\alpha}(z) \frac{\left(\mathcal{L}_{\alpha}^{k}(\varphi)\right)^{\prime}((z+1) / 2)}{2} d z .
$$

We focus on the first term above, the estimate for the second one being easier. We shall set $\bar{x}=x_{\ell}$, for suitable $\ell \geq 1$ to be determined below, where

$$
x_{\ell}:=\left(g_{\alpha}\right)^{\ell}(1) \leq 2^{1 / \alpha^{2}+1 / \alpha} \ell^{-1 / \alpha}
$$

by [LSV, Lemma 3.2]. Next, for every $1 \leq m \leq k$, setting $y_{m}\left(x_{\ell}\right)=\left(g_{\alpha}\right)^{m}\left(x_{\ell}\right)=x_{\ell+m}$, and $\phi=\phi_{k+1-m}-\rho_{\alpha}$, we have

$$
\begin{aligned}
\left\|\chi_{x>x_{\ell}}\left[\mathcal{L}_{\alpha}^{m}(\phi)\right]^{\prime}\right\|_{1} & \left.\leq\left\|\mathcal{L}_{\alpha}^{m}\left(\chi_{y>y_{m}}\left|\phi^{\prime}\right| /\left(T_{\alpha}^{m}\right)^{\prime}\right)\right\|_{1}+\| \mathcal{L}_{\alpha}^{m}\left(\chi_{y>y_{m}}\left|\phi \|\left(T_{\alpha}^{m}\right)^{\prime \prime}\right| /\left(T_{\alpha}^{m}\right)^{\prime}\right)^{2}\right) \|_{1} \\
& \leq\left\|\chi_{y>y_{m}}\left|\phi^{\prime}\right| \cdot\left|\left(T_{\alpha}^{m}\right)^{\prime}\right|^{-1}\right\|_{1}+\left\|\chi_{y>y_{m}}|\phi|\left|\left(T_{\alpha}^{m}\right)^{\prime \prime}\right|\left|\left(T_{\alpha}^{m}\right)^{\prime}\right|^{-2}\right\|_{1} .
\end{aligned}
$$

There exist $\lambda_{m}=\lambda_{m}\left(x_{\ell}\right)<1$ and $\Lambda_{m}\left(x_{\ell}\right)<\infty$ (both depending on $\alpha$ ) so that the first term in the right-hand side is $<\lambda_{m}\left\|\chi_{y>y_{m}(\ell)}\left|\phi^{\prime}\right|\right\|_{1}$ and the second term is $\leq \Lambda_{m}\|\phi\|_{1}$. In fact, we claim that there exists $C_{\alpha}>0$ so that for all $\ell$

$$
\lambda_{m}\left(x_{\ell}\right) \leq C_{\alpha}(1+m / \ell)^{-1-1 / \alpha} .
$$

Indeed, recalling that $f_{\alpha}=\left.T_{\alpha}\right|_{[0,1 / 2]}$, we have $\lambda_{m}\left(x_{\ell}\right)^{-1}=\left(f_{\alpha}^{m}\right)^{\prime}(y)$ for some $y \geq y_{m}\left(x_{\ell}\right)$, and bounded distortion 8 of $f_{\alpha}^{m}$ on $\left(y_{m}, f_{\alpha}\left(y_{m}\right)\right)=\left(y_{m}, y_{m-1}\right)$ gives

$$
\lambda_{m}\left(x_{\ell}\right) \leq C \frac{f_{\alpha}\left(y_{m}\right)-y_{m}}{f_{\alpha}\left(x_{\ell}\right)-x_{\ell}}=C \frac{y_{m}^{1+\alpha}}{x_{\ell}^{1+\alpha}} \leq C_{\alpha} \frac{1}{(1+m / \ell)^{1+1 / \alpha}},
$$

where we used the upper bound $y_{m}\left(x_{\ell}\right)=x_{\ell+m} \leq 2^{1 / \alpha^{2}+1 / \alpha}(\ell+m)^{-1 / \alpha}$ from (2.45) and the lower bound from $9\left[\mathrm{BT}\right.$, p. 606] (replacing their $x+x^{1+\alpha}$ by our $x+2^{\alpha} x^{1+\alpha}$ )

$$
x_{\ell} \geq c\left(2^{\alpha} \alpha\right)^{-\frac{1}{\alpha}} \ell^{-1 / \alpha} .
$$

Recalling that $\phi=\phi_{k+1-m}-\rho_{\alpha}$, we get, if $\alpha \in(0,1)$,

$$
\left\|\chi_{x>x_{\ell}}\left[\mathcal{L}_{\alpha}^{m}(\phi)\right]^{\prime}\right\|_{1} \leq \lambda_{m}\left(x_{\ell}\right)\left\|\chi_{y>y_{m}}\left(\left|\phi_{k+1-m}^{\prime}\right|+\left|\rho_{\alpha}^{\prime}\right|\right)\right\|_{1}+\Lambda_{m} C_{\alpha} \frac{(\log (k+1-m))^{1 / \alpha}}{(k+1-m)^{1 / \alpha-1}} .
$$

Recall that $\left|\phi_{\ell}^{\prime}(x)\right| \leq(a / x) \phi_{\ell} \leq C b_{1} x^{-\alpha-1}$ so that $\left\|\chi_{y>y_{m}}\left|\phi_{\ell}^{\prime}\right|\right\|_{1} \leq C y_{m}^{-\alpha}$ and $\left|\rho_{\alpha}^{\prime}(x)\right| \leq$ $c_{2} b_{1} x^{-\alpha-1}$, giving the same asymptotics, and note that (2.47) gives

$$
y_{m}\left(x_{\ell}\right) \geq c \alpha^{-\frac{1}{\alpha}}(m+\ell)^{-1 / \alpha}
$$

\footnotetext{
${ }^{8}$ See e.g. [LSV] (2) p. 678] for the bounded distortion property.

${ }^{9}$ Note that $\alpha+1$ should read $\alpha-1$ in line 7 of the proof of [BT] Prop. 2, p 606], that $+\alpha(\alpha+1) /\left(2 u_{n+1}\right)$ should be replaced by $-\alpha(\alpha-1) /\left(2 u_{n+1}\right)$ in line 8 , that $+\log n \cdot \alpha(\alpha+1) / 2$ should be replaced by $-\log ((1+\alpha n) /(1+\alpha)) \cdot(\alpha-1) / 2$ on line 10, and that $\log n \cdot \alpha(\alpha+1) /(2 n)$ should be replaced by $-\log (1+\alpha n) \cdot(\alpha-1) /(2 n)$ in line 12 .
} 
uniformly in $k$. Hence, using 2.47) for $y_{m}(\ell)=x_{m+\ell}$, we get

$$
\left\|\chi_{y>y_{m}\left(x_{\ell}\right)}\left(\left|\phi_{k+1-m}^{\prime}\right|+\left|\rho_{\alpha}^{\prime}\right|\right)\right\|_{1} \leq C \int_{y_{m}}^{1} y^{-1-\alpha} d y \leq C y_{m}^{-\alpha} \leq C \alpha(m+\ell) .
$$

Clearly, 2.46 implies

$$
C \alpha(m+\ell) \lambda_{m}\left(x_{\ell}\right) \leq C_{\alpha} \alpha \frac{m+\ell}{(1+m / \ell)^{1+1 / \alpha}} \leq C_{\alpha} \alpha \frac{\ell}{(1+m / \ell)^{1 / \alpha}} .
$$

Choosing first $\ell \geq 1$ to make 2.44) small, then $m \geq \ell$ to make 2.48 small, and finally taking $k \geq m$ large enough (i.e., $\beta$ close enough to $\alpha$ ) so that

$$
\Lambda_{m} C_{\alpha}(\log (k+1-m))^{1 / \alpha}(k+1-m)^{1-1 / \alpha}
$$

is small, proves $(2.42)$ in view of $(2.43)$ and 2.44$)$.

This proves the result for bounded $\psi$. If $\|\psi\|_{L^{q}}=1$ for $(1-\alpha)^{-1}<q<\infty$, we observe that $\operatorname{Leb}(\{\psi(x)>M\}) \leq M^{-q}$ and define $\psi_{M}(x)=\min (M, \psi(x))$, noting that $\left\|\psi-\psi_{M}\right\|_{L^{1}} \leq M^{1-q}$ and more generally $\left\|\psi-\psi_{M}\right\|_{L^{r}} \leq M^{1-q / r}$ for $r>1$ close to 1 . Since $|\log (2 x)| \in L^{r /(r-1)}(d x)$ for all $r>1$, we can generalise Steps 0 and 1 to $\psi \in L^{q}(d x)$ the result by taking $\epsilon>0$ very small and $r>1$ so that $q>r(1-\epsilon \alpha) /(1-\alpha-\epsilon \alpha)$ and choosing $\eta>0$ small enough so that $\frac{1}{(q / r)-1}<\eta<\frac{1}{\alpha}-\epsilon-1$, taking $M(j)=j^{\eta}$, and decomposing $\psi=\left(\psi-\psi_{M(j)}\right)+\psi_{M(j)}$ in the $j$ th term of (2.23), 2.35), and (2.34). We get two series each time. The first one is convergent because $\eta((q / r)-1)>1$ while the second one converges because $\eta+1<1 / \alpha-\epsilon$. For Step 2, we take $M(k)=k^{\eta}$ for $\eta \in(0,1 / \alpha-1)$ in (2.41).

Finally, the claim about continuity of the derivative follows from the linear response formula and our control on the tails of the absolutely convergent series therein.

\section{Appendix A. Proof of Propositions 2.4 and 2.6}

Proof of Proposition 2.4. The proof for the first derivative is similar to that of the proof of [LSV, Lemma 2.3, Lemma 5.1]. We concentrate on the statement for $\mathcal{N}_{\alpha}$, the proof for $\mathcal{L}_{\alpha}$ follows easily since $\left(\mathcal{L}_{\alpha}-\mathcal{N}_{\alpha}\right)(\varphi)(x)=\varphi((x+1) / 2) / 2$. Let $0 \leq \alpha<1$. We have $T_{\alpha}^{\prime}(x)=$ $1+2^{\alpha}(\alpha+1) x^{\alpha} \geq 1, T_{\alpha}^{\prime \prime}(x)=2^{\alpha}(\alpha+1) \alpha x^{\alpha-1} \geq 0$, and $T_{\alpha}^{\prime \prime \prime}(x)=2^{\alpha}(\alpha+1) \alpha(\alpha-1) x^{\alpha-2} \leq 0$. Throughout this proof, we set (recall (2.1p) $y=g_{\alpha}(x)$.

For $\varphi$ as in the statement of the proposition, we have (both terms are positive)

$$
\begin{aligned}
-\left(\mathcal{N}_{\alpha} \varphi\right)^{\prime}(x) & =\frac{T_{\alpha}^{\prime \prime}(y)}{\left(T_{\alpha}^{\prime}(y)\right)^{3}} \varphi(y)-\frac{1}{\left(T_{\alpha}^{\prime}(y)\right)^{2}} \varphi^{\prime}(y) \\
& \leq\left(\frac{T_{\alpha}^{\prime \prime}(y)}{\left(T_{\alpha}^{\prime}(y)\right)^{2}}+\frac{b_{1}}{y\left(T_{\alpha}^{\prime}(y)\right)}\right) \frac{\varphi(y)}{T_{\alpha}^{\prime}(y)} \\
& \leq \frac{b_{1}}{x}\left(\mathcal{N}_{\alpha} \varphi\right)(x) \sup _{y \in[0,1 / 2]}\left[\frac{T_{\alpha}(y)}{b_{1}} \cdot\left(\frac{T_{\alpha}^{\prime \prime}(y)}{\left(T_{\alpha}^{\prime}(y)\right)^{2}}+\frac{b_{1}}{y T_{\alpha}^{\prime}(y)}\right)\right] .
\end{aligned}
$$


Let $\Omega_{1}(y)$ be the term in square brackets, then we find if $b_{1} \geq 1+\alpha$

$$
\begin{aligned}
\Omega_{1}(y) & =\frac{T_{\alpha}(y)}{b_{1} y T_{\alpha}^{\prime}(y)}\left(\frac{y T_{\alpha}^{\prime \prime}(y)}{T_{\alpha}^{\prime}(y)}+b_{1}\right) \leq \frac{1+2^{\alpha} y^{\alpha}}{1+2^{\alpha}(1+\alpha) y^{\alpha}} \cdot\left(\frac{1}{b_{1}} \frac{2^{\alpha}(\alpha+1) \alpha y^{\alpha}}{1+2^{\alpha}(1+\alpha) y^{\alpha}}+1\right) \\
& =\left(1-\frac{2^{\alpha} \alpha y^{\alpha}}{1+2^{\alpha}(1+\alpha) y^{\alpha}}\right) \cdot\left(1+\frac{1}{b_{1}} \frac{2^{\alpha}(\alpha+1) \alpha y^{\alpha}}{1+2^{\alpha}(1+\alpha) y^{\alpha}}\right) \\
& \leq\left(1-\frac{2^{\alpha} \alpha y^{\alpha}}{1+2^{\alpha}(1+\alpha) y^{\alpha}}\right) \cdot\left(1+\frac{2^{\alpha} \alpha y^{\alpha}}{1+2^{\alpha}(1+\alpha) y^{\alpha}}\right),
\end{aligned}
$$

which is $\leq 1$ for all $y \in[0,1 / 2]$ (we used $(\alpha+1) / b_{1} \leq 1$ in the last line). Note that if $\alpha=0$ then $\Omega_{1}(y)=\frac{2}{b_{1}} \frac{b_{1}}{2} \equiv 1$. To get the reverse inequality, observe that $\bar{\Omega}_{1}(y)=$ $\frac{T_{\alpha}(y)}{y T_{\alpha}^{\prime}(y)}\left(\frac{y T_{\alpha}^{\prime \prime}(y)}{b_{1} T_{\alpha}^{\prime}(y)}+1\right) \geq 1$ if $\bar{b}_{1}>0$ is small enough (just revisit A.1P).

Next, writing $T$ instead of $T_{\alpha}$ for simplicity $\left|\left(\mathcal{N}_{\alpha} \varphi\right)^{\prime \prime}(x)\right|$ is bounded by (all terms below are nonnegative)

$$
\begin{aligned}
& -3 \frac{\varphi^{\prime}(y) T^{\prime \prime}(y)}{\left(T^{\prime}(y)\right)^{4}}-\frac{\varphi(y) T^{\prime \prime \prime}(y)}{\left(T^{\prime}(y)\right)^{4}}+3 \frac{\varphi(y)\left(T^{\prime \prime}(y)\right)^{2}}{\left(T^{\prime}(y)\right)^{5}}+\frac{\varphi^{\prime \prime}(y)}{\left(T^{\prime}(y)\right)^{3}} \\
& \leq \mathcal{N}_{\alpha} \varphi(x)\left(3\left(\frac{b_{1}}{y}\right) \frac{T^{\prime \prime}(y)}{\left(T^{\prime}(y)\right)^{3}}-\frac{T^{\prime \prime \prime}(y)}{\left(T^{\prime}(y)\right)^{3}}+3 \frac{\left(T^{\prime \prime}(y)\right)^{2}}{\left.T^{\prime}(y)\right)^{4}}+\left(\frac{b_{2}}{y^{2}}\right) \frac{1}{\left(T^{\prime}(y)\right)^{2}}\right) \\
& \leq \frac{b_{2}}{x^{2}} \mathcal{N}_{\alpha} \varphi(x)\left[\frac { T ( y ) ^ { 2 } } { b _ { 2 } | T ^ { \prime } ( y ) | ^ { 2 } } \left(3\left(\frac{b_{1}}{y}\right) \frac{2^{\alpha}(\alpha+1) \alpha y^{\alpha-1}}{T^{\prime}(y)}+\frac{2^{\alpha}(\alpha+1) \alpha|\alpha-1| y^{\alpha-2}}{T^{\prime}(y)}\right.\right. \\
& \left.\left.\quad+3 \frac{\left(2^{\alpha}(\alpha+1) \alpha y^{\alpha-1}\right)^{2}}{\left(T^{\prime}(y)\right)^{2}}+\left(\frac{b_{2}}{y^{2}}\right)\right)\right] .
\end{aligned}
$$

The term $\Omega_{2}(y)$ in square brackets can be written

$$
\frac{T(y)^{2}}{y^{2}\left|T^{\prime}(y)\right|^{2}}\left(1+\frac{2^{\alpha} \alpha y^{\alpha}}{\left(1+2^{\alpha}(\alpha+1) y^{\alpha}\right)} \frac{1}{b_{2}}\left[3 b_{1}(\alpha+1)+\left(1-\alpha^{2}\right)+3 \frac{2^{\alpha}(\alpha+1)^{2} \alpha y^{\alpha}}{1+2^{\alpha}(\alpha+1) y^{\alpha}}\right]\right) .
$$

We can fix $b_{2}>3 b_{1}(1+\alpha)+20$ large enough so that $\Omega_{2}(y) \leq 1$ for all $y \in[0,1 / 2]$ because

$$
\left(\frac{T(y)}{y T^{\prime}(y)}\right)^{2}=\left(\frac{1+2^{\alpha} y^{\alpha}}{1+2^{\alpha}(\alpha+1) y^{\alpha}}\right)^{2}=\left(1-\frac{2^{\alpha} \alpha y^{\alpha}}{1+2^{\alpha}(\alpha+1) y^{\alpha}}\right)^{2} .
$$

(Note that if $\alpha=0$ then $\Omega_{2}(y) \equiv 1$.) For the reverse inequality, observe that

$$
\begin{aligned}
\bar{\Omega}_{2}(y):= & \left(1-\frac{2^{\alpha} \alpha y^{\alpha}}{1+2^{\alpha}(\alpha+1) y^{\alpha}}\right)^{2} \\
& \cdot\left(1+\frac{2^{\alpha} \alpha y^{\alpha}}{\left(1+2^{\alpha}(\alpha+1) y^{\alpha}\right)} \frac{1}{\bar{b}_{2}}\left[3 \bar{b}_{1}(\alpha+1)+\left(1-\alpha^{2}\right)+3 \frac{2^{\alpha}(\alpha+1)^{2} \alpha y^{\alpha}}{1+2^{\alpha}(\alpha+1) y^{\alpha}}\right]\right) \geq 1,
\end{aligned}
$$

if $\bar{b}_{1} / \bar{b}_{2}>0$ is large enough.

Proof of Proposition 2.6. Note that $m(\varphi)=m\left(\mathcal{L}_{\alpha}(\varphi)\right)$ and $\mathcal{L}_{\alpha}\left(\rho_{\alpha}\right)=\rho_{\alpha}$. Also, $m(\varphi) \leq$ $2 m\left(\mathcal{N}_{\alpha}(\varphi)\right)$ (using our assumption) and $\mathcal{N}_{\alpha}\left(\rho_{\alpha}\right)<\rho_{\alpha}$. By A.1 we have $\mathcal{L}_{\alpha}\left(\mathcal{C}_{*, 1}(\alpha)\right) \subset$ $\mathcal{C}_{*, 1}\left(\alpha, a, b_{1}\right)$ and $\mathcal{N}_{\alpha}\left(\mathcal{C}_{*, 1}(\alpha)\right) \subset \mathcal{C}_{*, 1}\left(\alpha, 2 a, b_{1}\right)$. 
For the decay claim, use that $\mathcal{C}_{*}(0) \subset \mathcal{C}_{*}(\beta)$ for any $\beta \in(0,1)$, and fix $\beta=\alpha$. For $\epsilon>0$ we set ${ }^{10} \mathbb{A}_{\epsilon} \varphi(x)=(2 \epsilon)^{-1} \int_{y \in \mathcal{S}^{1}:|x-y|<\epsilon} \varphi(y) d y$. Then, revisiting [LSV, Proposition 3.3] for $\alpha=0$, we see that we can take $n_{\epsilon}$ there to be $|\log \epsilon| / \log 2$. Since [LSV, Lemma 3.1] implies $\left\|\mathcal{L}_{0}^{n_{\epsilon}}\left(\mathrm{id}-\mathbb{A}_{\epsilon}\right)(\varphi)\right\|_{1} \leq \frac{18 a b_{1} c_{2}}{\beta(1-\beta)}\|\varphi\|_{1}$ if $\varphi \in \mathcal{C}_{*, 1}\left(\beta, b_{1}\right)$, and since $\mathcal{C}_{*}(\alpha)$ is invariant under $\mathcal{L}_{0}$, the first paragraph of the proof of [LSV, Thm 4.1], taking $\epsilon=n^{-1 / \alpha}$ gives (2.18). (Note that [LSV, Lemma 2.4] is not needed when invoking [LSV, Prop 3.3] in the proof of [LSV, Thm 4.1] for $T_{0}$, since we may obtain an easier lower bound.)

\section{Appendix B. A CONE-ONLY PROOF FOR $\alpha \in[0,1 / 2)$ AND $\psi \in L^{\infty}$}

We show how to modify the proof of Theorem 2.1 to bypass the use of Gouëzel's results [Go, Goth] when $\alpha<1 / 2$ and $\psi \in L^{\infty}$ (exploiting only [LSV]).

We first note that in the setting of Proposition 2.6 there exists $C_{\alpha}=C_{\alpha}\left(a, b_{1}\right)>0$, with $\sup _{\beta \in[\alpha,(1+\alpha) / 2]} C_{\beta}\left(a, b_{1}\right)<\infty$, so that for any $\psi \in L^{\infty}$ and $\varphi \in \mathcal{C}_{*, 1}(\alpha)+\mathbb{R}$ with $\int \varphi d x=0$,

$$
\int_{0}^{1} \psi \mathcal{L}_{\alpha}^{k}(\varphi) d x \leq C_{\alpha}\|\psi\|_{L^{\infty}}\|\varphi\|_{L^{1}} \frac{(\log k)^{1 / \alpha}}{k^{\frac{1}{\alpha}-1}}, \forall k \geq 1
$$

Indeed, note that [LSV, Lemma 3.1] applies to $\mathcal{C}_{*, 1}$ instead of $\mathcal{C}_{*}$, up to replacing $10 a$ there by $18 a b_{1} c_{2}$. (In the computation, just use that $|\varphi(x)-\varphi(y)| \leq \sup _{z \in[x, y]}\left|\varphi^{\prime}(z)\right| \epsilon \leq$ $2 a b_{1} c_{2} \epsilon x^{-1-\alpha}$, if $|x-y| \leq \epsilon$ with $x \leq y$. The original 10 in [LSV] is obtained as $4 \times 2+2$ : the definition of our cone $\mathcal{C}_{*, 1}$ incorporates an additional factor of 2 , to make $4 \times 2 \times 2+2=18$ as well as introducing extra factor of $c_{2}$, as in (2.14), while the $b_{1}$ appears since we use the derivative of $\phi$, as just noted. Finally, apply the argument ${ }^{11}$ in the first paragraph of ${ }^{12}$ the proof of [LSV, Thm 4.1]. The proof gives $C_{\alpha}=\frac{36 a b_{1} c_{2}}{\alpha(1-\alpha)} 2^{(2+1 / \alpha)\left(\frac{1}{\alpha}-1\right)}\left(\frac{\frac{1}{\alpha}-1}{\gamma}\right)^{1 / \alpha}$, for some small $\gamma>0$. In particular, $C_{\alpha}$ becomes very large as $\alpha \rightarrow 0$. This ends the proof of (B.1).

We need to introduce the following cone:

$$
\mathcal{C}_{3}=\left\{\varphi \in \mathcal{C}^{3}(0,1]\left|\varphi \in \mathcal{C}_{2},\right| \varphi^{\prime \prime \prime}(x) \mid \leq \frac{b_{3}}{x^{3}} \varphi(x), \quad \forall x \in(0,1]\right\} .
$$

If $b_{3} \geq b_{1}$ is large enough then the invariance statements of Proposition 2.4 also hold for $\mathcal{C}_{3}$, indeed, noting that $T_{\alpha}^{(i v)}(x)=2^{\alpha}(\alpha+1) \alpha(\alpha-1)(\alpha-2) x^{\alpha-3} \geq 0$, we have

$$
\begin{aligned}
& \left|\left(\mathcal{N}_{\alpha} \varphi\right)^{\prime \prime \prime}(x)\right| \leq \frac{\left|\varphi^{\prime \prime \prime}(y)\right|}{\left|T^{\prime}(y)\right|^{4}}+6 \frac{\left|\varphi^{\prime \prime}(y) T^{\prime \prime}(y)\right|}{\left|T^{\prime}(y)\right|^{5}}+4 \frac{\left|\varphi^{\prime}(y) T^{\prime \prime \prime}(y)\right|}{\left|T^{\prime}(y)\right|^{5}}+15 \frac{\left|\varphi^{\prime}(y)\left(T^{\prime \prime}(y)\right)^{2}\right|}{\left|T^{\prime}(y)\right|^{6}} \\
& \quad+\frac{\left|\varphi(y) T^{(i v)}(y)\right|}{\left|T^{\prime}(y)\right|^{5}}+4 \frac{\left|\varphi(y) T^{\prime \prime \prime}(y)\right|}{\left|T^{\prime}(y)\right|^{6}}+6 \frac{\left|\varphi(y) T^{\prime \prime}(y) T^{\prime \prime \prime}(y)\right|}{\left|T^{\prime}(y)\right|^{6}}+15 \frac{\left|\varphi(y)\left(T^{\prime \prime}(y)\right)^{3}\right|}{\left|T^{\prime}(y)\right|^{7}}
\end{aligned}
$$

\footnotetext{
${ }^{10}$ Identifying $[0,1]$ with the circle $\mathcal{S}^{1}$.

${ }^{11}$ There is a typo there and one should take in fact $\epsilon=n^{-1 / \alpha}\left(2^{2+1 / \alpha} \gamma^{-1}\left(\frac{1}{\alpha}-1\right) \log n\right)^{1 / \alpha}$.

${ }^{12}$ Just like for [LSV], Prop. 5.4].
} 
and this is bounded by

$$
\begin{aligned}
\mathcal{N}_{\alpha} \varphi(x)\left(\frac{b_{3}}{y^{3}} \frac{1}{\left|T^{\prime}(y)\right|^{3}}+\frac{6 b_{2}}{y^{2}} \frac{1}{\left|T^{\prime}(y)\right|^{4}}+\frac{b_{1}}{y}\left(4 \frac{\left|T^{\prime \prime \prime}(y)\right|}{\left|T^{\prime}(y)\right|^{4}}+15 \frac{\left|\left(T^{\prime \prime}(y)\right)^{2}\right|}{\left|T^{\prime}(y)\right|^{5}}\right)\right. \\
\left.+\frac{\left|T^{(i v)}(y)\right|}{\left|T^{\prime}(y)\right|^{4}}+4 \frac{\left|T^{\prime \prime \prime}(y)\right|^{2}}{\left|T^{\prime}(y)\right|^{5}}+6 \frac{\left|T^{\prime \prime}(y) T^{\prime \prime \prime}(y)\right|}{\left|T^{\prime}(y)\right|^{5}}+15 \frac{\left|\left(T^{\prime \prime}(y)\right)^{3}\right|}{\left|T^{\prime}(y)\right|^{6}}\right) \\
\leq \frac{b_{3}}{x^{3}} \mathcal{N}_{\alpha} \varphi(x)\left[\frac { T ( y ) ^ { 3 } } { | T ^ { \prime } ( y ) | ^ { 3 } y ^ { 3 } } \left(1+\frac{6 b_{2} y}{b_{3}\left|T^{\prime}(y)\right|}+\frac{b_{1} y^{2}}{b_{3}}\left(4 \frac{\left|T^{\prime \prime \prime}(y)\right|}{\left|T^{\prime}(y)\right|}+15 \frac{\left|\left(T^{\prime \prime}(y)\right)^{2}\right|}{\left|T^{\prime}(y)\right|^{2}}\right)\right.\right. \\
\left.\left.+\frac{y^{3}}{b_{3}}\left(\frac{\left|T^{(i v)}(y)\right|}{\left|T^{\prime}(y)\right|}+4 \frac{\left|T^{\prime \prime \prime}(y)\right|^{2}}{\left|T^{\prime}(y)\right|^{2}}+6 \frac{\left|T^{\prime \prime}(y) T^{\prime \prime \prime}(y)\right|}{\left|T^{\prime}(y)\right|^{2}}+15 \frac{\left|\left(T^{\prime \prime}(y)\right)^{3}\right|}{\left|T^{\prime}(y)\right|^{3}}\right)\right)\right] .
\end{aligned}
$$

The term $\Omega_{3}(y)$ in square brackets is $\leq 1$ for all $y \in[0,1 / 2]$ if $b_{3}$ is large enough because

$$
\left(\frac{T(y)}{y T^{\prime}(y)}\right)^{3}=\left(\frac{1+2^{\alpha} y^{\alpha}}{1+2^{\alpha}(\alpha+1) y^{\alpha}}\right)^{3}=\left(1-\frac{2^{\alpha} \alpha y^{\alpha}}{1+2^{\alpha}(\alpha+1) y^{\alpha}}\right)^{3} .
$$

It is easy to see that $\rho_{\alpha} \in \mathcal{C}_{3}$, that $\mathcal{L}_{\alpha} \mathbf{1} \in \mathcal{C}_{*, 1}\left(\alpha, a, b_{1}\right) \cap \mathcal{C}_{3}$, etc. In fact, each occurrence of $\mathcal{C}_{2}$ in the proof of Theorem 2.1 can be replaced by $\mathcal{C}_{3}$.

We now go over the changes in the proof of Theorem 2.1. Consider first Step 0: If $\alpha \in(0,1 / 2)$ then, using (2.2), (2.3), (2.4), and (2.5), together with (2.12) and (2.17), and the fact that $\left|\left(\mathcal{N}_{\alpha}\left(\rho_{\alpha}\right)\right)^{\prime \prime}\right| \leq a c_{2} b_{2} x^{-2-\alpha}$, it is easy to see that there exists $a>0$ and a uniformly bounded constant $C_{X}>0$ so that $-\left(X_{\alpha}^{\prime} \mathcal{N}_{\alpha}\left(\rho_{\alpha}\right)\right)^{\prime}+C_{X}$ belongs to $\mathcal{C}_{*, 1}\left(\alpha, a, b_{1}\right)$, up to increasing $a$ and $b_{1}$ (see (2.13)). Next (B.1) applied to the zero-average function $\varphi=-\left(X_{\alpha} \mathcal{N}_{\alpha}\left(\rho_{\alpha}\right)\right)^{\prime} \in \mathcal{C}_{*, 1}+\mathbb{R}$ gives $C_{\alpha}>0$ so that the $j$ th term in the right-hand side of (2.23) is bounded by

$$
C_{\alpha} j^{1-1 / \alpha}(\log j)^{1 / \alpha}\|\psi\|_{L^{\infty}}\left\|\left(X_{\alpha} \mathcal{N}_{\alpha}\left(\rho_{\alpha}\right)\right)^{\prime}\right\|_{1} .
$$

Since $\alpha<1 / 2$, this is summable.

In Step 1, proceeding as in Step 0 in $\$ 2.3$ (using $(2.11)$ to invoke $(2.12)$ in order to get (2.17)), we find for any $1 \leq j \leq k-1$ a real constant $C_{j, k}<\infty$ so that

$$
\left\{ \pm X_{\alpha}^{\prime} \mathcal{N}_{\alpha}\left(\mathcal{L}_{\alpha}^{k-j-1}(\mathbf{1})\right)+C_{j, k}, X_{\alpha}\left[\mathcal{N}_{\alpha}\left(\mathcal{L}_{\alpha}^{k-j-1}(\mathbf{1})\right)\right]^{\prime}\right\} \subset \mathcal{C}_{1, *}\left(\alpha, 2 a, B_{1}\right) .
$$

Indeed, to show $(\mathrm{B} .2)$, setting $\left.\varphi=\mathcal{L}_{\alpha}^{k-j-1}(\mathbf{1})\right) \in \mathcal{C}_{*}$, and noting that $\varphi \geq 0$ and $\varphi^{\prime} \leq 0$, so that $\mathcal{N}_{\alpha} \varphi \geq 0$ and $\left(\mathcal{N}_{\alpha} \varphi\right)^{\prime} \leq 0$ so that $X_{\alpha}\left(\mathcal{N}_{\alpha} \varphi\right)^{\prime} \geq 0$ it is enough to check that

$$
\left|X_{\alpha}^{\prime \prime} \mathcal{N}_{\alpha}(\varphi)\right|(x)+\left|X_{\alpha}^{\prime}\left(\mathcal{N}_{\alpha}(\varphi)\right)^{\prime}\right|(x) \leq \frac{B_{1}}{2 x}\left|X_{\alpha}^{\prime}(x)\right| \mathcal{N}_{\alpha}(\varphi)(x),
$$

(which follows from $-\mathcal{N}_{\alpha}(\varphi)^{\prime}(x) \leq b_{1} \mathcal{N}_{\alpha}(\varphi)(x) / x$ and (2.4), (2.5)), and

$$
\left|X_{\alpha}^{\prime}\left(\mathcal{N}_{\alpha}(\varphi)\right)^{\prime}+X_{\alpha}\left(\mathcal{N}_{\alpha}(\varphi)\right)^{\prime \prime}\right|(x) \leq \frac{B_{1}}{2 x} X_{\alpha}(x)\left(\mathcal{N}_{\alpha}(\varphi)\right)^{\prime}(x)
$$

(which follows from $\mathcal{N}_{\alpha}(\varphi)^{\prime \prime}(x) \leq b_{2} \mathcal{N}_{\alpha}(\varphi)(x) / x^{2} \leq-b_{2} \mathcal{N}_{\alpha}(\varphi)^{\prime}(x) /\left(\bar{b}_{1} x\right)$ and (2.3), (2.4)). 
Next, if $0<\alpha<\beta<1 / 2$, by using $\mathcal{C}_{1, *}(\alpha) \subset \mathcal{C}_{1, *}(\beta)$ and $(\mathrm{B} .1)$, we get summability of the first term of the expression in the right-hand side of (2.34) as $k \rightarrow \infty$ :

$$
\left|\sum_{j=0}^{k-1} \int_{0}^{1} \psi \mathcal{L}_{\beta}^{j}\left(\left[X_{\alpha} \mathcal{N}_{\alpha}\left(\mathcal{L}_{\alpha}^{k-j-1}(\mathbf{1})\right)\right]^{\prime}\right) d x\right| \leq C_{\beta}\|\psi\|_{L^{\infty}} \sum_{j=0}^{k-1} \frac{(\log j)^{1 / \beta}}{j^{-1+1 / \beta}} .
$$

When we consider the second term of the right-hand side of (2.34), we require the derivatives of order three in $\mathcal{C}_{3}$. Applying $(2.33)$ to $\varphi=\mathcal{L}_{\alpha}^{k-j-1}(\mathbf{1}) \in \mathcal{C}_{3} \cap \mathcal{C}_{*, 1}(\alpha)$, and using Proposition 2.4, we see that for any $\alpha \leq \gamma \leq \beta$, up to taking larger $a$ and $b_{1}$ (uniformly in $1 \leq j \leq k-1$ ) the decomposition (2.37) of $\partial_{\gamma}^{2} \mathcal{L}_{\gamma}\left(\mathcal{L}_{\alpha}^{k-j-1}(\mathbf{1})\right.$ ) gives seven functions which, up to multiplying by -1 and adding a uniformly bounded constant, all lie in $\mathcal{C}_{*, 1}\left(\alpha, a, b_{1}\right) \subset \mathcal{C}_{*, 1}(\beta)$. We proceed as in the proof of $(\overline{\mathrm{B} .2})$, developing the Leibniz inequality. We shall focus on the contribution of $X_{\alpha}^{2}\left(\mathcal{N}_{\alpha}(\varphi)\right)^{\prime \prime}$, leaving the other terms to the reader. We need to check that

$$
\left|2 X_{\alpha} X_{\alpha}^{\prime}\left(\mathcal{N}_{\alpha}(\varphi)\right)^{\prime \prime}+X_{\alpha}^{2}\left(\mathcal{N}_{\alpha}(\varphi)\right)^{\prime \prime \prime}\right| \leq \frac{B_{1}}{7 x}\left|X_{\alpha}^{2}\left(\mathcal{N}_{\alpha}(\varphi)\right)^{\prime \prime}\right|
$$

The above bound follows from $\left(\mathcal{N}_{\alpha}(\varphi)\right)^{\prime \prime \prime}(x) \leq b_{3} \mathcal{N}_{\alpha}(\varphi)(x) / x^{3} \leq b_{3}\left(\mathcal{N}_{\alpha}(\varphi)\right)^{\prime \prime}(x) /\left(\bar{b}_{2} x\right)$ and (2.3), 2.4). Since we are in a cone, we may apply (B.1) once more, we thus get the bound

$$
\frac{\|\psi\|_{L^{\infty}}}{\beta-\alpha} \int_{\alpha}^{\beta}(\gamma-\alpha) C_{\beta} \sum_{j=0}^{k-1} \frac{(\log j)^{1 / \beta}}{j^{-1+1 / \beta}} d \gamma \leq C_{\beta}\|\psi\|_{L^{\infty}}(\beta-\alpha) .
$$

Step 2 does not change, and the proof of Theorem 2.1 bypassing [Go, Goth] is complete.

\section{REFERENCES}

[B1] V. Baladi, On the susceptibility function of piecewise expanding interval maps, Comm. Math. Phys. 275 (2007) 839-859.

[B2] V. Baladi, Linear response despite critical points, Nonlinearity 21 (2008) T81-T90.

[B3] V. Baladi, Linear response, or else, ICM Seoul 2014, Proceedings, Volume III, 525-545, http://www.icm2014.org/en/vod/proceedings .

[BMS] V. Baladi, S. Marmi, and D. Sauzin Natural boundary for the susceptibility function of generic piecewise expanding unimodal maps, Ergodic Theory Dynam. Systems 10 (2013) 1-24.

[BS] V. Baladi and D. Smania, Linear response formula for piecewise expanding unimodal maps, Nonlinearity 21 (2008) 677-711. (Corrigendum: Nonlinearity 25 (2012) 2203-2205).

[BCV] T. Bomfim, A. Castro, and P. Varandas, Differentiability of thermodynamical quantities in nonuniformly expanding dynamics, arXiv:1205.5361, to appear Adv. Math.

[BT] H. Bruin and M. Todd, Equilibrium states for potentials with $\sup \phi-\inf \phi<h_{\text {top }}(f)$, Comm. Math. Phys. 283 (2008) 579-611.

[CD] F. Contreras and D. Dolgopyat, Regularity of absolutely continuous invariant measures for piecewise expanding unimodal maps, arXiv:1504.04214.

[Do] D. Dolgopyat, On differentiability of SRB states for partially hyperbolic systems, Invent. Math. 155 (2004) 389-449.

[FT] J.M. Freitas and M. Todd, Statistical stability of equilibrium states for interval maps, Nonlinearity 22 (2009) 259-281.

[Go] S. Gouëzel, Sharp polynomial estimates for the decay of correlations, Israel J. Math. 139 (2004) 29-65. 
[Goth] S. Gouëzel, Vitesse de décorrélation et théorèmes limites pour les applications non uniformément dilatantes, $\mathrm{PhD}$ thesis, Orsay, 2004.

[HM] M. Hairer and A.J. Majda, A simple framework to justify linear response theory, Nonlinearity 23 (2010) 909-922.

[KKPW] A. Katok, G. Knieper, M. Pollicott, and H. Weiss, Differentiability and analyticity of topological entropy for Anosov and geodesic flows, Invent. Math. 98 (1989) 581-597.

[Ko] A. Korepanov, Linear response for intermittent maps with summable and non-summable decay of correlations, arXiv:1508.06571.

[LSV] C. Liverani, B. Saussol, and S. Vaienti, A probabilistic approach to intermittency, Ergodic Theory Dynam. Systems 19 (1999) 671-685.

[Lu1] V. Lucarini, D. Faranda, J. Wouters, and T. Kuna, Towards a general theory of extremes for observables of chaotic dynamical systems, J. Stat. Phys. 154 (2014) 723-750.

[Lu2] V. Lucarini et al., Extremes and Recurrence in Dynamical Systems, John Wiley and Sons, 2015.

[Ma] M. Mazzolena, Dinamiche espansive unidimensionali: dipendenza della misura invariante da un parametro, Master's Thesis, Roma 2 (2007).

[Sa] O. Sarig, Subexponential decay of correlations, Invent. Math. 150 (2002) 629-653.

[Ru] D. Ruelle, Differentiation of SRB states, Comm. Math. Phys. 187 (1997) 227-241.

[Ru1] D. Ruelle, General linear response formula in statistical mechanics, and the fluctuation-dissipation theorem far from equilibrium, Phys. Lett. A 245 (1998) 220-224.

[Ru2] D. Ruelle, Structure and $f$-dependence of the A.C.I.M. for a unimodal map $f$ of Misiurewicz type, Comm. Math. Phys., 287 (2009) 1039-1070.

[Ru3] D. Ruelle, A review of linear response theory for general differentiable dynamical systems, Nonlinearity 22 (2009) 855-870.

[Th1] M. Thaler, Estimates of the invariant densities of endomorphisms with indifferent fixed points, Israel J. Math. 37 (1980) 303-314.

[Th2] M. Thaler, The asymptotics of the Perron-Frobenius operator of a class of interval maps preserving infinite measures, Studia Math. 143 (2000) 103-119.

D.M.A., UMR 8553, École Normale Supérieure, 75005 Paris, France

Current address: Sorbonne Universités, UPMC Univ Paris 06, CNRS, Institut de Mathématiques de Jussieu-Paris Rive Gauche (IMJ-PRG), Analyse Algébrique, 4, Place Jussieu, 75005 Paris, France

E-mail address: viviane.baladi@imj-prg.fr

Mathematical Institute, University of St Andrews, North Haugh, St Andrews, KY16 9SS, SCOTLAND

E-mail address: m.todd@st-andrews.ac.uk 